TRABAJOS ORIGINALES

Rev Obstet Ginecol Venez. 2021; 81 (4): 319-328. https://doi.org/10.51288/00810405

\title{
Cáncer de endometrio: influencia del tratamiento en la calidad de vida y función sexual
}

\author{
(D) Ernesto Lara, ${ }^{1}$ (D) Franco Calderaro di Ruggiero. ${ }^{2}$
}

\begin{abstract}
RESUMEN
Objetivo: Determinar la influencia del tratamiento en la calidad de vida y función sexual de mujeres sobrevivientes de cáncer de endometrio durante el periodo: enero 2016 - agosto 2019.

Métodos: Investigación prospectiva, de tipo observacional, analítica, longitudinal, en sobrevivientes de cáncer de endometrio, a quienes se les aplicó el cuestionario de salud SF-12 y el Índice de Función Sexual Femenina, para determinar la calidad de vida y salud sexual respectivamente, antes y después del tratamiento.

Resultados: La frecuencia de pacientes expuestas a cirugía sola que percibieron deterioro en su salud fisica después del tratamiento fue 56,5\%, mientras que en las expuestas a cirugía más tratamiento adyuvante fue 97,4\%, en las expuestas a tratamiento neo adyuvante mas cirugía fue 100,0\% al igual que en las expuestas a radioterapia externa solamente. Ninguna paciente tratada con cirugía o radioterapia externa sola percibió deterioro en su salud mental después del tratamiento, mientras que las pacientes sometidas a cirugía más tratamiento adyuvante el porcentaje fue de 33,33\% y en las expuestas a tratamiento neo adyuvante mas cirugía fue 12,50\%. 40 mujeres presentaron disfunción sexual después del tratamiento, $65,5 \%$ de las mujeres sometidas a cirugía sola, 93,3\% de las sometidas a cirugía más tratamiento adyuvante y $100 \%$ de las expuestas a tratamiento neo adyuvante más cirugía.

Conclusión: La prevalencia de deterioro de la salud fisica, salud mental y disfunción sexual en las mujeres expuestas a tratamientos combinados fue superior que en las expuestas a cirugía sola.

Palabras clave: Cáncer de Endometrio, Calidad de vida, Función sexual, Salud física, Salud Mental.
\end{abstract}

\section{SUMMARY}

Objective: To determine the influence of the treatment received, in the quality of life and sexual function of women survivors of endometrial cancer during the period: January 2017 - August 2019.

Methods: Prospective, observational, analytical, longitudinal research, in endometrial cancer survivors, to whom the SF-12 health questionnaire and the Female Sexual Function Index were applied, to determine the quality of life and sexual health respectively, before and after treatment.

Results: Patients exposed to surgery alone who perceived deterioration in their physical health after treatment was $56.5 \%$, while in those exposed to surgery plus adjuvant treatment was $97.4 \%$, in those exposed to neoadjuvant treatment plus surgery was $100.0 \%$ as in those exposed to external radiotherapy only. No patient treated with surgery or external radiotherapy alone perceived deterioration in their mental health after treatment, while the patients undergoing surgery plus adjuvant treatment the percentage was $33.33 \%$ and in those exposed to neoadjuvant treatment plus surgery was $12.50 \% .40$ women had sexual dysfunction after treatment, $65.5 \%$ of women undergoing surgery alone, $93.3 \%$ of those undergoing surgery plus adjuvant treatment and $100 \%$ of those exposed to neoadjuvant treatment plus surgery.

Conclusion: The prevalence of deterioration of physical health, mental health and sexual dysfunction in women exposed to combined treatments was higher than in those exposed to surgery alone.

Key words: Endometrial Cancer, Quality of Life, Sexual Function, Physical Health, Mental Health.

\begin{abstract}
${ }^{1}$ Ginecólogo Oncólogo, Ginecólogo Obstetra. Adjunto del Servicio de Ginecología y Obstetricia, Hospital Dr. Domingo Luciani. Caracas, Venezuela. ${ }^{2}$ Doctor en Ciencias de la Salud, Cirujano General - Oncólogo, Ginecólogo Oncólogo, Jefe del Servicio y Director del Postgrado de Ginecología Oncológica (UCV), SOH - IVSS, Caracas, Venezuela. Correo de correspondencia: ernestolarav@gmail.com
\end{abstract}

Forma de citar este artículo: Lara E, Calderaro di Ruggiero F. Cáncer de endometrio: influencia del tratamiento en la calidad de vida y función sexual Rev Obstet Ginecol Venez. 2021; 81(4):319-328. https://doi. org/10.51288/00810405

\section{INTRODUCCIÓN}

El cáncer de endometrio (CE) representa el sexto trastorno maligno más común en la población femenina a nivel mundial. Se estima que anualmente se diagnostican 382069 nuevos casos, teniendo los países de altos ingresos una mayor incidencia de CE, 


\section{CÁNCER DE ENDOMETRIO: INFLUENCIA DEL TRATAMIENTO \\ EN LA CALIDAD DE VIDA Y FUNCIÓN SEXUAL}

en comparación con los países de bajos recursos (1).

En EE. UU., representa el cáncer ginecológico más frecuente, con un estimado de 61880 nuevos casos y 12160 muertes en 2019 (2). En Venezuela, ocupa la sexta causa de incidencia por cáncer en la población femenina, con una incidencia de 951 casos anuales, con un pico máximo entre los 55 y 67 años de edad (3).

Aunque convencionalmente se piensa que el cáncer de endometrio es una neoplasia del periodo posmenopáusico, el $14 \%$ de los casos se diagnostican en mujeres premenopáusicas, el $5 \%$ de las cuales son menores de 40 años (4-6). El principal factor de riesgo es la exposición a estrógenos endógenos y exógenos, asociados con la obesidad, diabetes, edad temprana de la menarquia, nuliparidad, menopausia tardía, edad avanzada ( $\geq 55$ años) y el uso de tamoxifeno (7-12).

La mayoría de las mujeres son diagnosticadas en estadios iniciales y la cirugía es el tratamiento primario, incluyendo histerectomía total, salpingooforectomía bilateral y estadificación quirúrgica, la cual puede incluir linfadenectomía pélvica y/o paraaórtica (13). Desde 1988, la International Federation of Gynecology and Obstetrics (FIGO), estableció un cambio significativo en la estadificación del CE, que previamente se hacía sobre la base de los hallazgos clínicos y pasó a realizarse quirúrgicamente, ya que en pacientes con estadio I de la enfermedad, los ganglios linfáticos son el lugar hacia donde más frecuentemente se disemina el tumor y la identificación del estatus ganglionar permite conocer qué pacientes pueden beneficiarse más de un tratamiento adyuvante $(14,15)$.

Aunque las tasas de supervivencia, la toxicidad, la respuesta tumoral o el funcionamiento físico son ampliamente medidos y han sido el enfoque principal al juzgar la eficacia de las terapias contra el cáncer, recientemente ha habido un creciente impulso para incorporar la calidad de vida (CV) y la función sexual
(FS) como rutina, tanto en el manejo clínico como en las investigaciones y ensayos clínicos (16). Las mejores tasas de sobrevida global así como la duración de la sobrevida, han impulsado dentro de la atención oncológica, el cambio de paradigma, de un modelo de enfermedad médica a un modelo de bienestar que involucre tanto la CV y la FS de las mujeres (17).

La CV se ha convertido, en los últimos años, en un objetivo importante a ser alcanzado en el área de la salud. Su evaluación es considerada como una nueva estrategia introducida para el análisis de los resultados de programas terapéuticos (18). Por otro lado, el bienestar sexual es uno de los factores primordiales en la sensación de placer de la mujer, ya que logra unificar la satisfacción física-emocional, además de fortalecer su propia identidad y sentido de feminidad, elementos claves en la percepción de la CV. Por ello, en la actualidad el funcionamiento sexual de las mujeres debe ser analizado de forma independiente, dada la importancia que representa dentro de la CV $(19,20)$.

Diferentes investigaciones clínicas han considerado la CV y la FS como objetivo principal. Li y cols. (21) determinaron en sobrevivientes de CE, una CV menor al compararlas con un grupo control. Zhu y cols. (22) indicaron que la radioterapia empeora significativamente la $\mathrm{CV}$ de las pacientes sometidas a cirugía estadificadora. Por otra parte, Aerts y cols. (23), demostraron que, en comparación con los controles sanos, las pacientes con $\mathrm{CE}$, tienen un alto riesgo de disfunciones sexuales, tanto antes como después del tratamiento quirúrgico.

En Venezuela, no hay estudios que definan el impacto en la CV y en la FS de las diferentes modalidades terapéuticas en pacientes con $\mathrm{CE}$, por lo tanto, el objetivo del estudio fue determinar la influencia que tiene el tratamiento, en la CV y en la FS, en mujeres supervivientes de CE. 


\section{MÉTODOS}

Se realizó una investigación prospectiva, de tipo observacional, analítica, con un diseño longitudinal. La población estuvo conformada por el total de mujeres supervivientes al CE, atendidas en la consulta de Ginecología Oncológica del "Servicio Oncológico Hospitalario del IVSS" de Caracas, durante el periodo enero 2016 - agosto 2019. Las pacientes se dividieron en grupos de acuerdo al tratamiento recibido: cirugía, cirugía más tratamiento adyuvante, radioterapia neoadyuvante más cirugía. Se obtuvo una muestra de 103 pacientes que cumplieron los siguientes criterios: diagnóstico confirmado de $\mathrm{CE}$, tener como mínimo 12 meses de haber culminado el tratamiento y estar libre de enfermedad. Se excluyeron pacientes con diagnóstico de psicosis o retraso mental.

Se procedió a seleccionar de manera no aleatoria e intencional en la consulta de Ginecología Oncológica, a las pacientes supervivientes al CE, de acuerdo con los criterios previamente establecidos. A cada paciente en el área de consulta, se le realizó el llenado de la ficha de trabajo y una encuesta con base en los instrumentos: Cuestionario de Salud SF-12 V2 (SF-12) y el Índice de Función Sexual Femenina (IFSF), previa explicación del procedimiento a seguir; así mismo, se le realizó una evaluación médica ginecológica integral, donde por medio de la entrevista clínica, se procedió a registrar los datos clínicos.

Los datos fueron tratados con técnicas propias de la estadística descriptiva y los resultados aquí presentados se circunscriben a la muestra. Las variables numéricas con nivel de medida de escala se describieron en términos de su media $(\overline{\mathrm{X}})$, desviación estándar (DE), percentiles y coeficiente de variación (CV) que indica la variación relativa y permite comparar la dispersión entre los grupos. Las variables categóricas se describieron en términos de frecuencias absolutas y relativas. Los datos se presentaron en cuadros y gráficos de caja que permiten comparar las distribuciones de las puntuaciones de los cuestionarios SF-12 V2 e IFSF antes y después del tratamiento de CE.

El punto de corte para evaluar la $\mathrm{CV}$ es 50 ; de tal forma que las puntuaciones $\leq 50$ son indicativas de deterioro en la $\mathrm{CV}$ relacionada con la salud mientras que puntuaciones superiores a 50 indican buena $\mathrm{CV}$ relacionada a la salud.

El punto de corte para evaluar la función sexual es 26,55 de tal manera que puntuaciones $<26,55$ son indicativas de disfunción sexual mientras que puntuaciones $\geq 26,55$ indican función sexual normal.

Como medidas de asociación se utilizó el coeficiente de correlación eta $\eta$ (que oscila entre 0 y 1 , donde 0 indica que no hay asociación y 1 que hay una asociación perfecta), indicado para medir la fuerza de la relación entre tratamiento (variable dicotómica) y puntuaciones del IFSF y del SF-12 V2 (variables cuantitativas). Todo el tratamiento estadístico se apoyó en el programa informático SPSS Statistics 19.

\section{RESULTADOS}

Se trata de una muestra intencional de 103 mujeres supervivientes de $\mathrm{CE}$, con más de doce meses de haber culminado el tratamiento, a las que se les aplicaron dos cuestionarios, destinados a recopilar información sobre la FS (Índice de función sexual femenina - IFSF) y CV (Cuestionario de Salud SF-12 V2).

Calidad de vida y tratamiento de cáncer de endometrio

En la dimensión salud física, la comparación de las distribuciones de las puntuaciones pretratamiento y postratamiento, reflejó un desplazamiento hacia la baja de esta última. Antes del tratamiento, 3 mujeres 


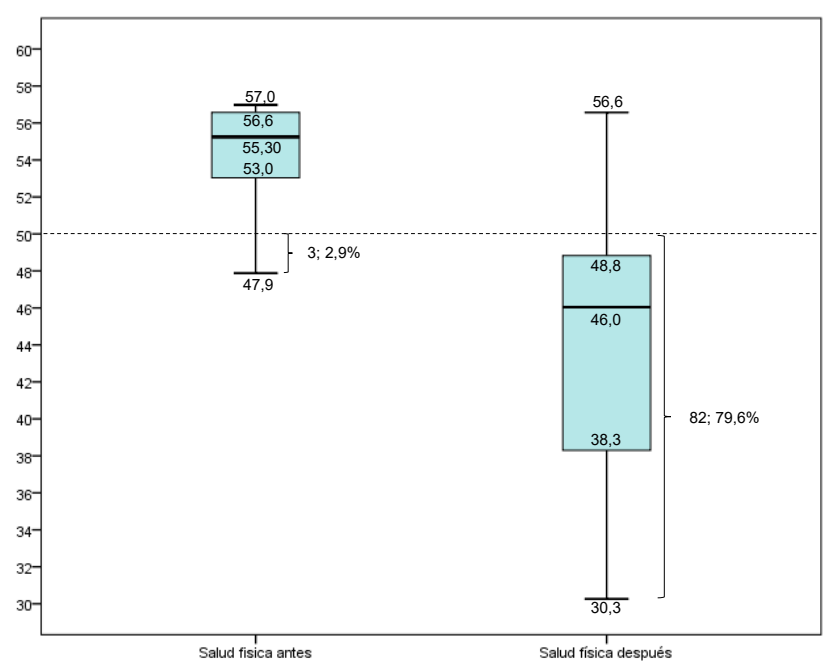

Gráfico 1. Cuestionario de Salud SF-12 V2:

Dimensión salud física. Puntuaciones de mujeres sobrevivientes a $\mathrm{CE}$

$(2,91 \%)$ percibieron deterioro en su salud física y 82 mujeres $(79,61 \%)$ lo percibieron después del mismo (Gráfico 1).

En la dimensión salud mental, la comparación de las distribuciones de las puntuaciones pretratamiento y postratamiento, también reflejó un desplazamiento hacia la baja de esta última. Antes del tratamiento, ninguna mujer percibió deterioro en su salud mental, mientras que 15 mujeres $(14,56 \%)$, lo percibieron después del mismo (Gráfico 2). Al comparar las dos dimensiones, la salud mental presentó menor deterioro que la salud física.

Con relación al tratamiento recibido, 46 mujeres $(44,66 \%)$ fueron expuestas a cirugía sola, 39 mujeres $(37,86 \%)$, recibieron cirugía más tratamiento adyuvante, 2 mujeres $(1,94 \%)$ recibieron radioterapia externa sola y 16 mujeres $(15,53 \%)$, recibieron tratamiento neoadyuvante más cirugía.

El porcentaje de pacientes expuestas a cirugía sola que percibieron deterioro en su salud física después del tratamiento, fue $56,5 \%$, mientras que en las pacientes expuestas a cirugía más tratamiento adyuvante fue

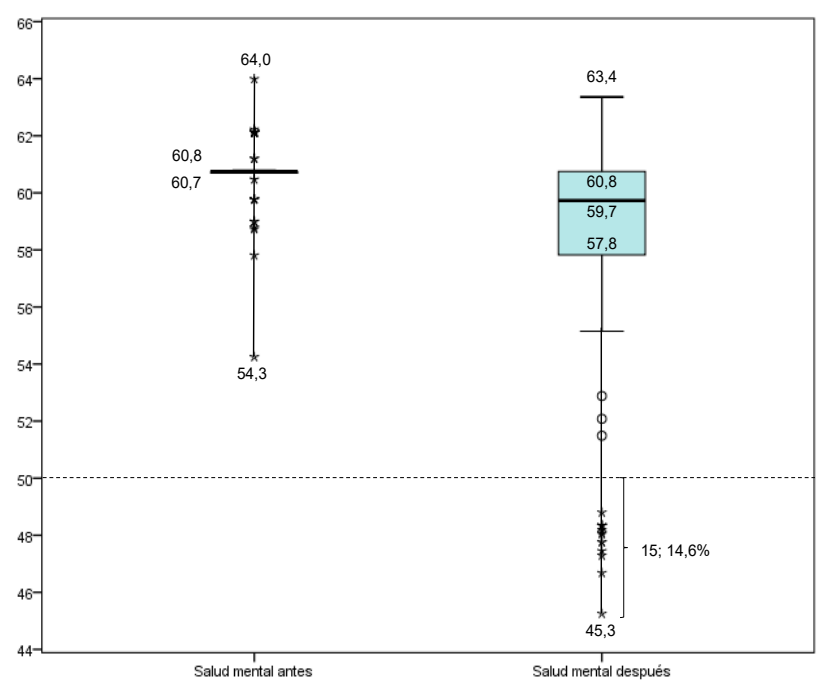

Gráfico 2. Cuestionario de Salud SF-12 V2: Dominio salud mental Puntuaciones de mujeres sobrevivientes a $\mathrm{CE}$

$97,4 \%$, en las expuestas a tratamiento neoadyuvante mas cirugía fue $100 \%$, al igual que en las expuestas a radioterapia externa (RTE) (Gráfico 3).

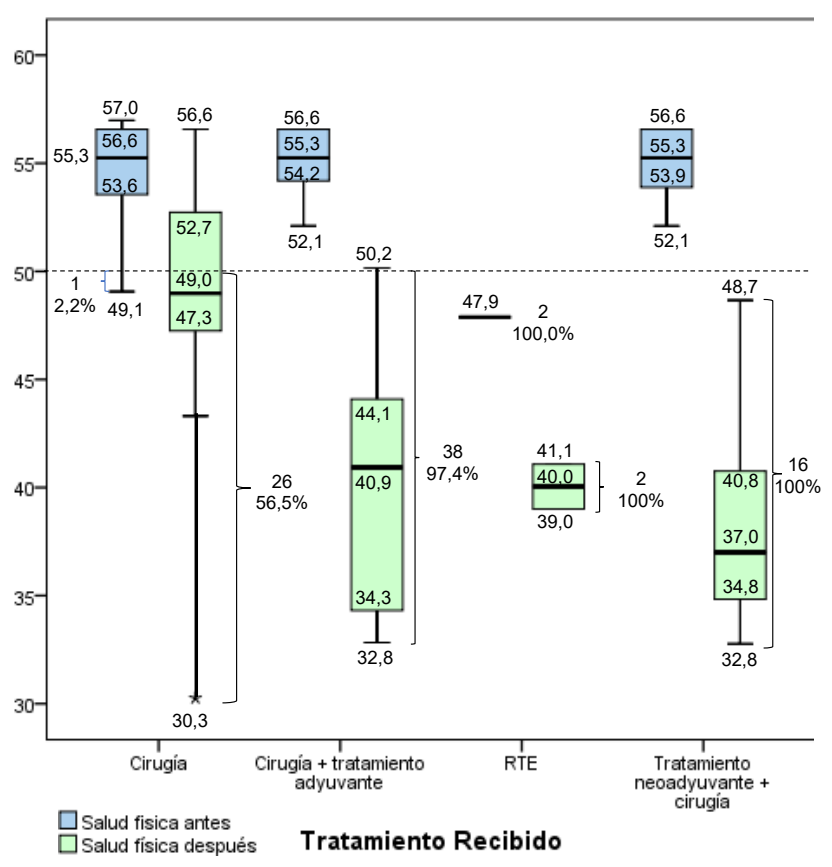

Gráfico 3. Cuestionario de Salud SF-12 V2:

Dimensión salud física. Puntuaciones de mujeres sobrevivientes a CE por tipo de tratamiento 
Con relación a la salud mental, ninguna paciente expuesta a cirugía sola, percibió deterioro en su salud mental después del tratamiento, mientras que en las pacientes expuestas a cirugía más tratamiento adyuvante, el porcentaje fue de 33,33 \% y en las expuestas a tratamiento neoadyuvante más cirugía, fue $12,50 \%$ (Gráfico 4).

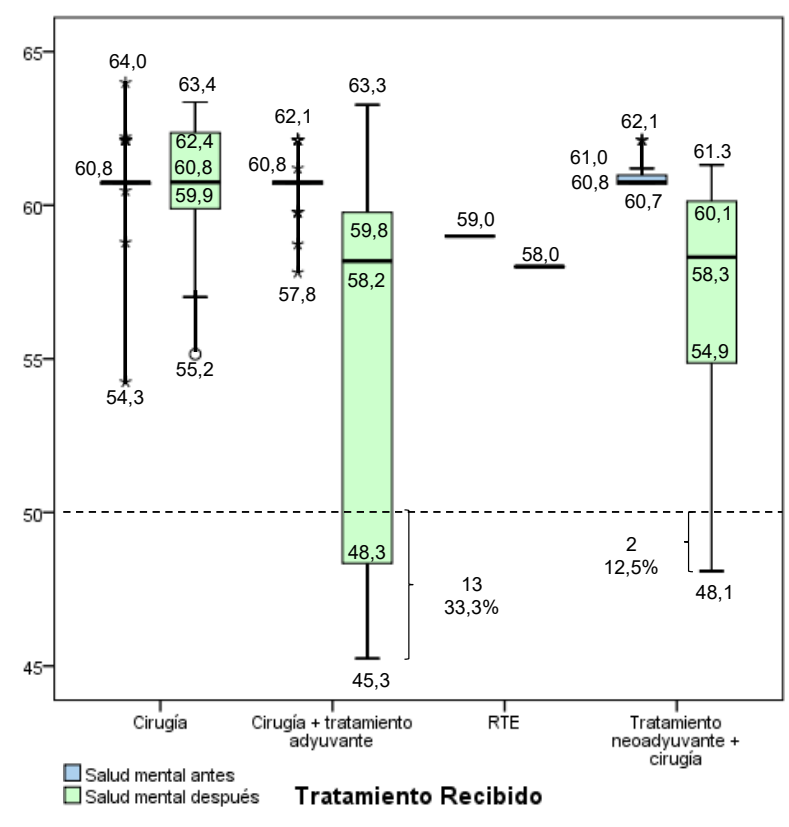

Gráfico 4. Cuestionario de Salud SF-12 V2:

Dimensión salud mental. Puntuaciones de mujeres sobrevivientes a $\mathrm{CE}$ por tipo de tratamiento

Los resultados expuestos, sugieren la existencia de cierto nivel de asociación entre el tipo de tratamiento y la calidad de vida percibida por las sobrevivientes de CE. Los valores del coeficiente de correlación eta para las relaciones salud mental/tratamiento $(0,53)$ y salud física/tratamiento $(0,73)$, indicaron que en ambos casos el grado de asociación fue moderado.

Función sexual femenina y tratamiento de cáncer de endometrio

De las 103 mujeres que integraron la muestra original, 51 respondieron el cuestionario IFSF, 52 (50,5\%) no lo hicieron porque manifestaron no tener pareja sexual, por lo que no fueron incluidas en el estudio de la función sexual femenina.

Se determinó que antes del tratamiento, $62,75 \%$ de las pacientes ya se encontraban en la categoría de disfunción sexual y $37,25 \%$, tenían un funcionamiento saludable. El porcentaje de mujeres con disfunción sexual se incrementó a 78,43\%, posterior al tratamiento. La comparación de las distribuciones de las puntuaciones pretratamiento y postratamiento reflejó un desplazamiento hacia la baja de esta última (Gráfico 5).

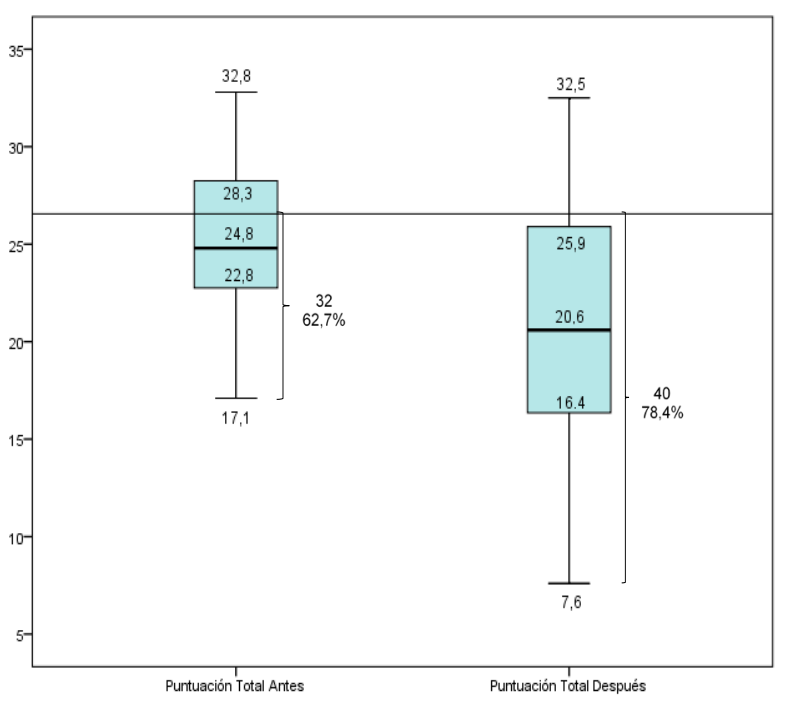

Gráfico 5. Escala general del IFSF. Puntuaciones de pacientes con pareja sexual antes y después del tratamiento de $\mathrm{CE}$

Aunque todos los dominios del IFSF presentaron deterioro, el dolor fue el aspecto en el que más mujeres lo percibieron y equivalen a $62,75 \%$ de la muestra. Le siguió excitación con 47,06 \% (Tabla 1).

Con relación al tratamiento recibido, la comparación de las distribuciones de puntuaciones de la escala general IFSF en el grupo expuesto a cirugía sola, frente a los 


\section{CÁNCER DE ENDOMETRIO: INFLUENCIA DEL TRATAMIENTO \\ EN LA CALIDAD DE VIDA Y FUNCIÓN SEXUAL}

Tabla 1. Número de mujeres agrupadas por categoría de cambio en la puntuación IFSF

\begin{tabular}{lcc}
\hline \multirow{2}{*}{ Sub escala } & \multicolumn{2}{c}{ Categorías de cambio } \\
\cline { 2 - 3 } & \multicolumn{2}{c}{$\mathrm{n}=51$} \\
\cline { 2 - 3 } & Deterioro & Sin cambio \\
\hline Deseo & $19(37,3)$ & $32(62,7)$ \\
Excitación & $24(47,1)$ & $27(52,9)$ \\
Lubricación & $22(43,1)$ & $29(56,9)$ \\
Orgasmo & $22(43,1)$ & $29(56,9)$ \\
Satisfacción & $18(35,3)$ & $33(64,7)$ \\
Dolor & $32(62,7)$ & $19(37,3)$ \\
Escala general & $34(66,7)$ & $17(33,3)$ \\
\hline
\end{tabular}

grupos expuestos a cirugía más tratamiento adyuvante o tratamiento neoadyuvante más cirugía, reflejó el desplazamiento hacia la baja de los tratamientos combinados. Las puntuaciones $<26,55$ que indicaban

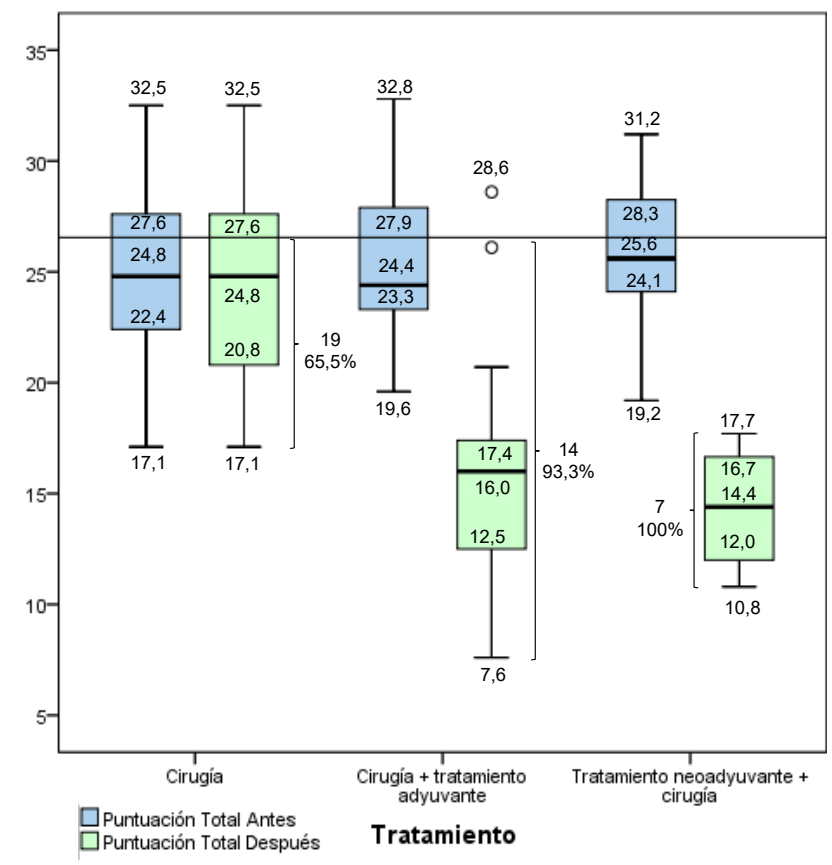

Gráfico 6. Escala General IFSF. Puntuaciones de mujeres con pareja sexual antes y después del tratamiento de CE clasificadas por tipo de tratamiento riesgo de disfunción sexual, fueron obtenidas en $65,5 \%$ expuestas a cirugía sola, en 93,3 \% expuestas a cirugía más tratamiento adyuvante y en el $100 \%$ expuestas a tratamiento neoadyuvante más cirugía (Gráfico 6).

Los resultados expuestos sugieren la existencia de cierto nivel de asociación entre el tipo de tratamiento y la FS percibida por las sobrevivientes de CE. En la escala general, el valor del coeficiente de correlación eta $(0,6999)$, indicó que el grado de asociación entre tratamiento y función sexual fue moderado.

\section{DISCUSIÓN}

Más del $75 \%$ de las pacientes con cáncer de endometrio son diagnosticadas en estadios iniciales, con tasas de supervivencia general a los 5 años de alrededor del $80 \%(24,25)$. Debido a los periodos de supervivencia a largo plazo, los problemas de CV se vuelven extremadamente importantes en estas pacientes. Por otro lado, se ha informado que el número de mujeres afectadas por alguna forma de disfunción sexual femenina, como menos deseo sexual, disminución de la capacidad para llegar al clímax y trastorno de dolor sexual, es sustancialmente mayor en pacientes con cáncer ginecológico que en mujeres sanas $(26,27)$. Sin embargo, la prevalencia de la disfunción sexual en las sobrevivientes de $\mathrm{CE}$ no ha sido bien documentada. Por lo tanto, se realizó este estudio utilizando dos cuestionarios, destinados a recopilar información sobre la función sexual femenina (IFSF) y la calidad de vida (SF-12 V2).

Las medias de las puntuaciones del cuestionario de salud SF 12 en el presente estudio, disminuyeron después del tratamiento, $80 \%$ de las mujeres percibió deterioro en la salud física y $15 \%$ lo percibió en la salud mental; esto es similar a lo reportado por Li y cols. (21) quienes determinaron que, en las sobrevivientes de $\mathrm{CE}$, la $\mathrm{CV}$ fue menor que en los controles, con una mayor prevalencia de síntomas somáticos. 
Van de Poll y cols. (28) señalaron que pacientes con cáncer de endometrio tratadas con cirugía, tienen una mejor $\mathrm{CV}$ que mujeres tratadas con cirugía y radioterapia externa. Tales datos son similares a los reportados en el presente estudio, donde las puntuaciones medias de ambas dimensiones del cuestionario de salud SF-12 en los grupos expuestos a tratamientos combinados, fueron inferiores a las del grupo expuesto a cirugía sola, apreciándose una asociación moderada entre tratamiento y salud física y mental.

Por otro lado, Klee y cols. (29) describieron la CV relacionada con la salud de pacientes con $\mathrm{CE}$ que están libres de enfermedad después de someterse a radioterapia externa y encontraron que la $\mathrm{CV}$ fue inferior a la de una población similar de mujeres sanas. $\mathrm{Al}$ igual que Zhu y cols. (22) quienes observaron que la radioterapia empeoró significativamente la CV de las pacientes sometidas a cirugía estadificadora. Esto contrasta con Le T y cols. (30) quienes no señalaron efectos adversos significativos de la radioterapia adyuvante sobre la $\mathrm{CV}$ en pacientes sobrevivientes de CE. En conformidad con esto, Karabuga y cols. (31) determinaron que la radioterapia externa afecta la calidad de vida a largo plazo en sobrevivientes de cáncer de endometrio.

Los datos de seguimiento de 13,3 años del ensayo PORTEC-1, mostraron que las pacientes tratadas con radioterapia externa, desarrollaron tasas más altas de incontinencia urinaria, diarrea y fuga fecal que condujeron a limitaciones en la vida diaria (32). En el ensayo PORTEC-2 posterior, que aleatorizó a las pacientes a tener radioterapia externa o braquiterapia, las pacientes con braquiterapia informaron un mejor funcionamiento social y escalas de síntomas más bajas en comparación con radioterapia externa (33). De manera similar, Sorbe y cols. (34), en un estudio prospectivo aleatorizado que comparó la radioterapia externa y la braquiterapia, demostraron que la radioterapia externa disminuyó el estado de salud global y el funcionamiento físico, de roles y social durante y después del tratamiento.

La principal diferencia del presente estudio con respecto a las investigaciones antes mencionadas, es que en este trabajo se compararon las diferentes modalidades propuestas para el manejo del CE, incluyendo la combinación de radioterapia externa y braquiterapia y/o el uso de quimioterapia.

Gao y cols. (35) señalaron que la quimioterapia afectó negativamente la $\mathrm{CV}$ en las pacientes sobrevivientes de CE. Del mismo modo, en este estudio se encontró mayor deterioro en la $\mathrm{CV}$, tanto en la salud física como en la salud mental (en menor porcentaje), en pacientes sometidas a tratamientos con quimioterapia y/o radioterapia, neoadyuvante o adyuvante.

Los factores que ayudan a explicar este mayor deterioro de la $\mathrm{CV}$ en grupos expuestos a tratamientos combinados, se centran en el mayor daño a los diferentes tejidos y aumento de la morbilidad, cuando se utiliza más de una modalidad tratamiento.

Con respecto a la FS, se ha reportado que el número de mujeres con cáncer ginecológico que son sexualmente activas, es significativamente menor que el número de mujeres sanas sexualmente activas (36). En el presente estudio, 41 pacientes $(39,8 \%)$ manifestaron no tener pareja sexual y 11 pacientes $(17,74 \%)$ no tuvieron relaciones sexuales posterior al tratamiento. De manera similar Gao y cols. (35) reportaron que $55,9 \%$ de sobrevivientes con CE manifestaron no tener relaciones sexuales. Onujiogu y cols. (37) señalaron que $69 \%$ de las pacientes sobrevivientes de CE tienen pareja sexual. Preocupaciones en las pacientes atribuidas a un posible debilitamiento en la potencia del tratamiento y el temor a la recurrencia del cáncer, se han informado como principales causas de inactividad sexual (35). 


\section{CÁNCER DE ENDOMETRIO: INFLUENCIA DEL TRATAMIENTO \\ EN LA CALIDAD DE VIDA Y FUNCIÓN SEXUAL}

Los resultados obtenidos reflejan el descenso de las puntuaciones después del tratamiento de CE; antes del tratamiento 32 mujeres $(62,75 \%)$ ya se encontraban en la categoría de disfunción sexual. El número de mujeres con disfunción sexual se incrementó a $40(78,43 \%)$ con la adición de 8 mujeres que anteriormente tenían un funcionamiento sano y bajaron su puntuación después del tratamiento. Esto se asemeja a los resultados de Gao y cols. (35) quienes encontraron que $68,6 \%$ de las sobrevivientes con CE tenían un IFSF $<26$, siendo diagnosticadas con disfunción sexual. Igualmente, Onujiogu y cols. (37) reportaron un $89 \%$ de disfunción sexual.

Aunque todos los dominios del IFSF presentaron deterioro, el dolor fue el más frecuentemente afectado ya que se presentó en $62,75 \%$ de los casos, seguido por la excitación, lubricación y orgasmo. Aerts y cols. (23), en comparación con mujeres sanas, encontraron que más mujeres con $\mathrm{CE}$, informaron disfunción sexual, incluyendo disfunción del deseo sexual, disfunción de excitación, dispareunia y una intensidad reducida del orgasmo.

En este estudio las mujeres expuestas a tratamientos combinados presentaron mayor prevalencia de disfunción sexual, $46 \%$ superior que las que fueron expuestas a cirugía sola, reflejándose una asociación moderada entre tratamiento y función sexual. Karabuga y cols. (31) señalaron que la radioterapia externa afectó negativamente la FS en sobrevivientes de $\mathrm{CE}$, en concordancia con Gao y cols. (35). En contraposición, Becker y cols. (38) encontraron que después de la cirugía, la braquiterapia adyuvante no tiene un impacto significativo en la función sexual.

A medida que más mujeres sobrevivan al cáncer de endometrio debido al avance médico, también deberían avanzar las estrategias para mejorar la $\mathrm{CV}$ y la FS, aspectos importantes para muchas pacientes sobrevivientes de cáncer; para lo cual es necesario una evaluación y comprensión precisa del problema.

Se concluye que:

Las medias de las puntuaciones del cuestionario de salud SF 12, disminuyeron después del tratamiento. La salud mental presentó menor deterioro que la salud física.

El descenso de las puntuaciones después del tratamiento del $\mathrm{CE}$, tanto de la escala general del IFSF, como de las 6 subescalas que la componen, determinaron un deterioro de la función sexual entre las mujeres evaluadas.

Las puntuaciones medias de ambas dimensiones del cuestionario de salud SF-12 en los grupos expuestos a tratamientos combinados, son inferiores a las del grupo expuesto a cirugía sola, apreciándose una asociación moderada entre tratamiento y salud física y mental.

Las mujeres expuestas a tratamientos combinados, presentaron mayor prevalencia de disfunción sexual, que las que fueron expuestas a cirugía sola, reflejándose una asociación moderada entre tratamiento y función sexual.

Los autores recomiendan realizar una evaluación precisa de la CV y la FS en las mujeres sobrevivientes de $\mathrm{CE}$, a fin de diagnosticar oportunamente el deterioro de las mismas, facilitando así el desarrollo de intervenciones dirigidas a corregirlas.

\section{REFERENCIAS}

1. Bray F, Ferlay J, Soerjomataram I, Siegel RL, Torre LA, Jemal A. Global cancer statistics 2018: GLOBOCAN estimates of incidence and mortality worldwide for 36 cancers in 185 countries. CA Cancer J Clin. 2018; 68(6):394-424. doi: 10.3322/caac.21492. 
2. Siegel RL, Miller KD, Jemal A. Cancer statistics, 2019. CA Cancer J Clin. 2019; 69(1):7-34. doi: 10.3322/ caac. 21551.

3. Capote Negrín L. Resumen del cáncer en Venezuela. Rev Venez Oncol [Internet]. 2015 [consultado 05/09/2020]; 27(4):256-268. Disponible en: http://www.oncologia.org. ve/site/upload/revista/pdf/10._capote_1_(256-268).pdf

4. Evans-Metcalf ER, Brooks SE, Reale FR, Baker SP. Profile of women 45 years of age and younger with endometrial cancer. Obstet Gynecol. 1998; 91(3):349354. doi: 10.1016/s0029-7844(97)00668-6.

5. Lee TS, Jung JY, Kim JW, Park NH, Song YS, Kang SB, et al. Feasibility of ovarian preservation in patients with early stage endometrial carcinoma. Gynecol Oncol. 2007; 104(1):52-57. doi: 10.1016/j.ygyno.2006.07.003.

6. Duska LR, Garrett A, Rueda BR, Haas J, Chang Y, Fuller AF. Endometrial cancer in women 40 years old or younger. Gynecol Oncol. 2001; 83(2):388-393. doi: 10.1006/gyno.2001.6434.

7. Key TJ, Pike MC. The dose-effect relationship between 'unopposed' oestrogens and endometrial mitotic rate: its central role in explaining and predicting endometrial cancer risk. Br J Cancer. 1988; 57(2):205-212. doi: 10.1038/bjc.1988.44.

8. Pike MC, Peters RK, Cozen W, Probst-Hensch NM, Felix JC, Wan PC, et al. Estrogen-progestin replacement therapy and endometrial cancer. J Natl Cancer Inst. 1997; 89(15):1110-1116. doi: 10.1093/jnci/89.15.1110.

9. Kaaks R, Lukanova A, Kurzer MS. Obesity, endogenous hormones, and endometrial cancer risk: a synthetic review. Cancer Epidemiol Biomarkers Prev [Internet]. 2002 [consultado 18 de agosto de 2019]; 11(12):153143. Disponible en: https://cebp.aacrjournals.org/ content/cebp/11/12/1531.full.pdf

10. Renehan AG, Tyson M, Egger M, Heller RF, Zwahlen M. Body-mass index and incidence of cancer: a systematic review and meta-analysis of prospective observational studies. Lancet. 2008; 371(9612):569578. doi: 10.1016/S0140-6736(08)60269-X.

11. Grady D, Gebretsadik T, Kerlikowske K, Ernster V, Petitti D. Hormone replacement therapy and endometrial cancer risk: a meta-analysis. Obstet Gynecol. 1995; 85(2):304-313. doi: 10.1016/0029-7844(94)00383-O.

12. Purdie DM, Green AC. Epidemiology of endometrial cancer. Best Pract Res Clin Obstet Gynaecol. 2001; 15(3):341-354. doi: 10.1053/beog.2000.0180.

13. National Comprehensive Cancer Network, NCCN Clinical Practice Guidelines in Oncology ( $\mathrm{NCCN}$
Guidelines) [Internet]. Uterine Neoplasms (Version 3.2019); 2019 [consultado: 18/08/19] https://www. nccn.org/professionals/physician_gls/pdf/uterine.pdf.

14. Shepherd JH. Revised FIGO staging for gynaecological cancer. Br J Obstet Gynaecol. 1989; 96(8):889-892. doi: 10.1111/j.1471-0528.1989.tb03341.x.

15. Ackerman I, Malone S, Thomas G, Franssen E, Balogh J, Dembo A. Endometrial carcinoma--relative effectiveness of adjuvant irradiation vs therapy reserved for relapse. Gynecol Oncol. 1996; 60(2):177-183. doi: 10.1006/gyno.1996.0022.

16. Jones GL, Ledger W, Bonnett TJ, Radley S, Parkinson N, Kennedy SH. The impact of treatment for gynecological cancer on health-related quality of life (HRQoL): a systematic review. Am J Obstet Gynecol. 2006; 194(1):26-42. doi: 10.1016/j.ajog.2005.04.060.

17. Zeng YC, Ching SS, Loke AY. Quality of life measurement in women with cervical cancer: implications for Chinese cervical cancer survivors. Health Qual Life Outcomes. 2010; 8:30. doi: 10.1186/1477-7525-8-30.

18. Ramírez R. Calidad de vida relacionada con la salud como medida de resultados en salud: revisión sistemática de la literatura. Rev Col Cardiol [Internet]. 2007 [consultado 18/08/2019]; 14(4):207-222. Disponible en: http://www.scielo.org.co/scielo.php?script=sci arttext\&pid=S0120-56332007000400004\&lng=en.

19. Botros SM, Abramov Y, Miller JJ, Sand PK, Gandhi S, Nickolov A, et al. Effect of parity on sexual function: an identical twin study. Obstet Gynecol. 2006; 107(4):765770. doi: 10.1097/01.AOG.0000207677.03235.76.

20. Komura LA, Herrmann P, Silva de Mello D, Massumi M. Histerectomía y vivencia de la sexualidad. Index Enferm [Internet]. 2007 [consultado 18/08/2019]; 16(57): 28-32. Disponible en: http://scielo.isciii.es/scielo.php?script=sci_ arttext\&pid=S1132-12962007000200006\&lng=es.

21. Li C, Samsioe G, Iosif C. Quality of life in endometrial cancer survivors. Maturitas. 1999; 31(3):227-236. doi: 10.1016/s0378-5122(98)00106-6.

22. Zhu L, Le T, Popkin D, Olatunbosun O. Quality-oflife analysis in the management of endometrial cancer. Am J Obstet Gynecol. 2005; 192(5):1388-1390. doi: 10.1016/j.ajog.2004.12.081.

23. Aerts L, Enzlin P, Verhaeghe J, Poppe W, Vergote I, Amant F. Sexual functioning in women after surgical treatment for endometrial cancer: a prospective controlled study. J Sex Med. 2015; 12(1):198-209. doi: 10.1111/jsm. 12764 . 


\section{CÁNCER DE ENDOMETRIO: INFLUENCIA DEL TRATAMIENTO \\ EN LA CALIDAD DE VIDA Y FUNCIÓN SEXUAL}

24. Blake P, Swart AM, Orton J, Kitchener H, Whelan T, Lukka H, et al.; ASTEC/EN.5 Study Group. Adjuvant external beam radiotherapy in the treatment of endometrial cancer (MRC ASTEC and NCIC CTG EN.5 randomised trials): pooled trial results, systematic review, and meta-analysis. Lancet. 2009; 373(9658):137-146. doi: 10.1016/S01406736(08)61767-5.

25. Hayat MJ, Howlader N, Reichman ME, Edwards BK. Cancer statistics, trends, and multiple primary cancer analyses from the Surveillance, Epidemiology, and End Results (SEER) Program. Oncologist. 2007; 12(1):2037. doi: 10.1634/theoncologist.12-1-20.

26. Ratner ES, Foran KA, Schwartz PE, Minkin MJ. Sexuality and intimacy after gynecological cancer. Maturitas. 2010; 66(1):23-26. doi: 10.1016/j. maturitas.2010.01.015.

27. Rutledge TL, Heckman SR, Qualls C, Muller CY, Rogers RG. Pelvic floor disorders and sexual function in gynecologic cancer survivors: a cohort study. Am J Obstet Gynecol. 2010; 203(5):514.e1-7. doi: 10.1016/j. ajog.2010.08.004.

28. van de Poll-Franse LV, Mols F, Essink-Bot ML, Haartsen $\mathrm{JE}$, Vingerhoets AJ, Lybeert ML, et al. Impact of external beam adjuvant radiotherapy on health-related quality of life for long-term survivors of endometrial adenocarcinoma: a population-based study. Int $\mathrm{J}$ Radiat Oncol Biol Phys. 2007; 69(1):125-132. doi: 10.1016/j. ijrobp.2007.02.040.

29. Klee M, Machin D. Health-related quality of life of patients with endometrial cancer who are diseasefree following external irradiation. Acta Oncol. 2001; 40(7):816-824. doi: 10.1080/02841860152703436.

30. Le T, Menard C, Samant R, Choan E, Hopkins L, Faught W, et al. Longitudinal assessments of quality of life in endometrial cancer patients: effect of surgical approach and adjuvant radiotherapy. Int $\mathrm{J}$ Radiat Oncol Biol Phys. 2009; 75(3):795-802. doi: 10.1016/j. ijrobp.2008.11.018.

31. Karabuga H, Gultekin M, Tulunay G, Yuce K, Ayhan A, Yuce D, et al. Assessing the Quality of Life in Patients With Endometrial Cancer Treated With Adjuvant Radiotherapy. Int J Gynecol Cancer. 2015; 25(8):15261533. doi: 10.1097/IGC.0000000000000509.
32. Nout RA, van de Poll-Franse LV, Lybeert ML, WárlámRodenhuis CC, Jobsen JJ, Mens JW, et al. Long-term outcome and quality of life of patients with endometrial carcinoma treated with or without pelvic radiotherapy in the post-operative radiation therapy in endometrial carcinoma 1 (PORTEC-1) trial. J Clin Oncol. 2011; 29(13):1692-1700. doi: 10.1200/JCO.2010.32.4590.

33. Nout RA, Putter H, Jürgenliemk-Schulz IM, Jobsen JJ, Lutgens LC, van der Steen-Banasik EM, et al. Five-year quality of life of endometrial cancer patients treated in the randomised Post-Operative Radiation Therapy in Endometrial Cancer (PORTEC-2) trial and comparison with norm data. Eur J Cancer. 2012; 48(11):1638-1648. doi: 10.1016/j.ejca.2011.11.014.

34. Sorbe BG, Horvath G, Andersson H, Boman K, Lundgren C, Pettersson B. External pelvic and vaginal irradiation versus vaginal irradiation alone as postoperative therapy in medium-risk endometrial carcinoma: a prospective, randomized study--qualityof-life analysis. Int J Gynecol Cancer. 2012; 22(7):1281 1288. doi: 10.1097/IGC.0b013e3182643ba0.

35. Gao H, Xiao M, Bai H, Zhang Z. Sexual Function and Quality of Life Among Patients With Endometrial Cancer After Surgery. Int J Gynecol Cancer. 2017; 27(3):608-612. doi: 10.1097/IGC.0000000000000905.

36. Grimm D, Hasenburg A, Eulenburg C, Steinsiek L, Mayer S, Eltrop S, et al. Sexual Activity and Function in Patients With Gynecological Malignancies After Completed Treatment. Int J Gynecol Cancer. 2015; 25(6):1134-1141. doi: 10.1097/ IGC.0000000000000468.

37. Onujiogu N, Johnson T, Seo S, Mijal K, Rash J, Seaborne L, et al. Survivors of endometrial cancer: who is at risk for sexual dysfunction? Gynecol Oncol. 2011; 123(2):356-359. doi: 10.1016/j.ygyno.2011.07.035.

38. Becker M, Malafy T, Bossart M, Henne K, Gitsch G, Denschlag D. Quality of life and sexual functioning in endometrial cancer survivors. Gynecol Oncol. 2011; 121(1):169-173. doi: 10.1016/j.ygyno.2010.11.024.

Recibido 4 de julio de 2021 Aprobado 25 de julio de 2021 